\title{
Macrophage to Leukocyte Ratio
}

National Cancer Institute

\section{Source}

National Cancer Institute. Macrophage to Leukocyte Ratio. NCI Thesaurus. Code

C123460.

The determination of the ratio of macrophages compared to leukocytes in a sample.

The measurement may be expressed as a ratio or percentage. 